\title{
EFEITO DOS ISOLADOS DE Metarhizium anisopliae (METSCHNIKOFF) SOROKIN (HYPOCREALES: CLAVICIPITACEAE) SOBRE PARASITOIDE Cotesia flavipes (CAMERON, 1891) (HYMENOPTERA: BRACONIDAE)
}

HAYASHIDA, Eduardo Kenji ${ }^{1}$

KASSAB, Samir Oliveira ${ }^{2}$ FONSECA, Paulo Rogério Beltramin da ${ }^{3}$

ROSSONI, Camila ${ }^{4}$

LOUREIRO, Elisângela de Souza ${ }^{5}$ AMORIM, Luis Gustavo Pessoa ${ }^{6}$

RESUMO: O objetivo deste trabalho foi avaliar o efeito dos isolados de Metarhizum anisopliae sobre o parasitoide larval Cotesia flavipes. O experimento foi conduzido à temperatura de $25 \pm 1^{\circ} \mathrm{C}$, UR de $70 \pm 10 \%$ e fotofase de $12 \mathrm{~h}$, empregando-se o delineamento inteiramente casualizado com 16 tratamentos e 5 repetições, com um total de 10 adultos de $C$. flavipes por repetição. Foram utilizados os seguintes tratamentos: Testemunha (não-tratada), isolados UFGD 05, IBCB 348 e IBCB 425 nas concentrações de $1.0 \times 10^{7} ; 0.5 \times 10^{8} ; 1.0 \times 10^{8} ; 0.5 \times 10^{9}$ e $1.0 \times 10^{9}$ conídios.mL $\mathrm{L}^{-1}$. Os insetos foram acondicionadas em tubos de plástico tipo Falcon ${ }^{\otimes}$, contendo um pedaço de papel filtro inoculado com $1 \mathrm{~mL}$ de suspensão fúngica e uma gota de mel. Os resultados demonstraram que os isolados UFGD 05, IBCB 348 e IBCB 425 apresentaram baixa virulência aos adultos do parasitóide $C$. flavipes.

Palavras-chave: Cana-de-açúcar. Controle microbiano. Entomopatógenos.

SUMMARY: The objective of this study was to evaluate the effect of isolated Metarhizum anisopliae on larval parasitoid Cotesia flavipes. The experiment was conducted at a temperature of $25 \pm 1{ }^{\circ} \mathrm{C}$, RH $70 \pm 10 \%$ and $12 \mathrm{~h}$ photophase, using a completely randomized design with 16 treatments and five repetitions, with a total of 10 adults C. flavipes by repetition. We used the following treatments: control (untreated), isolates UFGD 05, IBCB 348 and

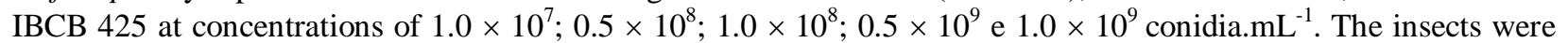
placed in plastic tubes of type Falcon ${ }^{\circledR}$, containing a piece of filter paper inoculated with $1 \mathrm{~mL}^{-1}$ of fungal suspension and a gout of honey. The results showed that the isolates UFGD 05, IBCB 348 and IBCB 425 had low virulence to adult parasitoids $C$. flavipes.

Keywords: Sugar cane. Microbial control. Entomopathogens.

\section{INTRODUÇÃO}

A cana-de-açúcar representa um dos principais produtos agrícolas do Brasil, o plantio da cultura é expandido a cada ano e atualmente a área cultivada ocupa aproximadamente 8 milhões de hectares do território nacional (CONAB, 2011).

\footnotetext{
${ }^{1}$ Biólogo, Mestrando em Entomologia e Conservação da Biodiversidade, Faculdade de Ciências Biológicas e Ambientais, UFGD. Rodovia Dourados-Itahum, Km 12, Caixa Postal 533, Bairro Aeroporto, CEP: 79804-970, Dourados-MS, e-mail: ekhayashida@hotmail.com.

${ }^{2}$ Biólogo, Doutorando em Entomologia e Conservação da Biodiversidade, Faculdade de Ciências Biológicas e Ambientais, UFGD. Rodovia Dourados-Itahum, Km 12, Caixa Postal 533, Bairro Aeroporto, CEP: 79804-970, Dourados-MS, e-mail: samirkassab@gmail.com.

${ }^{3}$ Eng ${ }^{\circ}$ Agrônomo, Doutorando em Agronomia (Produção Vegetal), Faculdade de Ciências Agrárias, UFGD. Rodovia DouradosItahum, Km 12, Caixa Postal 533, Bairro Aeroporto, CEP: 79804-970, Dourados-MS, e-mail: prbeltramin@ hotmail.com.

${ }^{4}$ Bióloga, Mestranda em Entomologia e Conservação da Biodiversidade, Faculdade de Ciências Biológicas e Ambientais, UFGD. Rodovia Dourados-Itahum, Km 12, Caixa Postal 533, Bairro Aeroporto, CEP: 79804-970, Dourados-MS, e-mail: camilarossoni15@hotmail.com;

${ }^{5} E_{n}$ a , Agr. Dra. Professora Adjunto I. Universidade Federal de Mato Grosso do Sul, Campus de Chapadão do Sul. Caixa Postal 112, CEP: 79560-000, Chapadão do Sul, MS, e-mail: elisangela.loureiro@ufms.br.

${ }^{6}$ Eng $^{\circ}$ Agrônomo, Professor Adjunto I. Universidade Federal de Mato Grosso do Sul, Campus de Chapadão do Sul. Caixa Postal 112, CEP: 79560-000, Chapadão do Sul, MS, e-mail: gugamorim@yahoo.com.br.
} 
Apesar da sua importância a cultura da cana-de-açúcar pode sofrer injúrias de muitos insetos, tornando-se fator limitante a produção sucroalcooleira. Dentre as espécies pragas, a de maior importância é a Diatraea saccharalis (Fabricius, 1794) (Lepidoptera: Crambidae), devido à ampla distribuição e dimensão dos prejuízos que causam (SILVA et al., 2010).

As lagartas de D. saccharalis alimentam-se dos tecidos foliares nos primeiros dias após a emergência da planta e no terceiro instar adentra o colmo da cana promovendo injúria direta (PINTO et al., 2009). A penetração dos fungos fitopatogênicos é favorecida indiretamente pelo inseto, causando o sintoma da podridão vermelha do colmo e queda na produção de açúcar e álcool (SENÔ et al., 2010).

Um dos métodos utilizados para o controle da D. saccharalis é o biológico. O inimigo natural Cotesia flavipes (Cameron, 1891) (Hymenoptera: Braconidae) têm se mostrado eficiente regulador desta lagarta, principalmente por uma crescente tendência na diminuição do uso de produtos químicos na agricultura (JIALE et al., 2010).

Os fungos entomopatogênicos podem ser utilizados no combate de pragas da cultura cana-deaçúcar sendo outro importante agente de controle biológico (TIAGO et al., 2011). São 90 gêneros e mais de 700 espécies de fungos entomopatogênicos, destacando-se a espécie Metarhizium anisopliae (Metschnikoff) Sorokin (Hypocreales: Clavicipitaceae) que há muito tempo é estudada e utilizada para controlar a cigarrinha da cana-de-açúcar Mahanarva fimbriolata (Stål, 1854) (Hemiptera: Cercopidae) (LOUREIRO et al., 2005).

O controle biológico nas culturas tem como finalidade manter as espécies de pragas em níveis tecnicamente aceitáveis através da introdução de um predador natural, parasitoide ou microrganismo que lhe cause doença ou morte (DINIZ et al., 2008). Essa tecnologia para o controle de insetos é utilizada há muito tempo e tornou-se interessante por razões ambientais e econômicas (MACEDO et al., 2009). No entanto, pesquisas que esclareçam a coexistência do entomopatógeno $M$. anisopliae e $C$. flavipes no mesmo ambiente devem ser efetuadas para que se verifique o efeito do fungo sobre o parasitoide.

O presente trabalho teve como objetivo verificar o efeito dos isolados de M. anisopliae sobre adultos de $C$. flavipes, em laboratório.

\section{MATERIAL E MÉTODOS}

O experimento foi realizado no laboratório de Microbiologia da Faculdade de Ciências Biológicas e Ambientais (FCBA) da Universidade Federal da Grande Dourados (UFGD). O ensaio experimental composto por um delineamento inteiramente casualizado (DIC) contendo a testemunha (não tratada), 15 tratamentos com 5 repetições e 10 adultos de $C$. flavipes recém de emergidos por repetição. As suspensões de conídios, utilizadas no experimento, foram obtidas a partir de colônias jovens dos isolados IBCB 425, IBCB 348 e UFGD 05 de M. anisopliae.

Para a montagem do bioensaio, dos isolados de M. anisopliae, foram preparadas suspensões em solução de Tween $80^{\circledR}$ a $0,1 \%$ nas concentrações de $1,0 \times 10^{7} ; 0,5 \times 10^{8} ; 1,0 \times 10^{8} ; 0,5 \times 10^{9}$ e $1,0 \times 10^{9}$ conídios. $\mathrm{mL}^{-1}$ aferidas mediante quantificação em câmara de Neubauer ${ }^{\circledR}$ com o auxílio de um microscópio óptico (ALVES et al., 1998). Os adultos de C. flavipes foram expostos ao fungo M. anisopliae em tubos de plástico tipo Falcon ${ }^{\circledR}$ contendo uma porção de papel filtro inoculado com $1 \mathrm{~mL}$ de suspensão e uma gota de mel na parede do tubo com a função de nutrir o parasitóide durante a condução do ensaio experimental. $\mathrm{Na}$ testemunha, o papel filtro foi tratado com água destilada esterilizada contendo Tween $80^{\circledR}$ a 0,1 e na parede do tubo inoculada uma gota de mel. Os tubos com os insetos foram mantidos em câmara climatizada a temperatura de $25 \pm 2^{\circ} \mathrm{C}$, umidade relativa de $70 \pm 10 \%$ e fotofase de 12 horas.

Avaliou-se a mortalidade diariamente, durante 12 dias após a aplicação (DAA) dos fungos entomopatogênicos.Cada inseto morto foi transferido para placa de Petri com algodão hidrófilo umedecido 
com água destilada esterilizada e novamente acondicionados em câmara climatizada para verificar da mortalidade confirmada.

A variável avaliada foi à mortalidade do parasitóide $C$. flavipes em porcentagem (\%). Foi utilizada a estatística descritiva para a interpretação dos resultados (GOMES, 1982).

\section{RESULTADOS E DISCUSSÃO}

De acordo com os dados amostrados, adultos do parasitóide $C$. flavipes expostos ao fungo IBCB 425 morrem logo no $7^{\circ}$ DAA. O isolado nas concentrações de $1,0 \times 10^{9}$ e $0,5 \times 10^{9}$ conídios. $\mathrm{mL}^{-1}$ causou $100 \%$ de mortalidade nos adultos do parasitóide $C$. flavipes (Tabela 1). Os dados não corrobam com os obtidos por Grance et al., (2009) os autores avaliaram o efeito de seis isolados do fungo Beauveria bassiana (Balsamo) Vuillemin, 1912 (Ascomycetes: Clavicipitaceae) sobre adultos do parasitóide Trichospilus diatraeae (Cherian \& Margabandhu, 1942) (Hymenoptera: Eulophidae) e observaram que os isolados UFGD $10\left(1,0 \times 10^{7}\right.$ e $0,5 \times 10^{8}$ conídios.mL $\left.{ }^{-1}\right)$, UFGD $06\left(1,0 \times 10^{7}\right.$ e $0,5 \times 10^{8}$ conídios.mL $\left.\mathrm{mL}^{-1}\right)$ e ESALQ $446\left(1,0 \times 10^{9}\right.$ e $0,5 \times 10^{8}$ conídios. $\left.\mathrm{mL}^{-1}\right)$ causaram menor efeito sobre $T$. diatraeae.

No tratamento com o isolado IBCB 348 observou-se que logo no $6^{\circ}$ DAA na concentração de 1,0 x $10^{7}$ conídios. $\mathrm{mL}^{-1}$ foi capaz de provocar $100 \%$ de mortalidade nos adultos do parasitóide C. flavipes (Tabela 1). Os resultados do presente estudo corroboram com os encontrados por Loureiro et al., (2005) os autores relataram que o isolado IBCB 348 apresentaram mortalidade confirmada de $80 \%$ para as ninfas de M. fimbriolata tratadas com a concentração de 5,0 x $10^{7}$ conídios. $\mathrm{mL}^{-1}$, ao sexto dia da aplicação (Tabela $1)$.

O isolado UFGD 05 causou mortalidade de $100 \%$ dos indivíduos aos $9^{\circ}$ DAA, na concentração de $0,5 \times 10^{8}$ conídios. $\mathrm{mL}^{-1}$ e as concentrações de $0,5 \times 10^{9}, 1,0 \times 10^{8}$ e $1,0 \times 10^{7}$ causaram mortalidade de $100 \%$ aos $10^{\circ} \mathrm{DAA}$, sendo que a dose de $1,0 \times 10^{8}$ obteve a maior mortalidade no $8^{\circ}$ DAA (Tabela 1 ). Em estudos conduzidos por Loureiro et al., (2007) os isolados de M. anisopliae foram patogênicos ao predador $O$. insidiosus, com 100\% de mortalidade confirmada aos 14 dias após a infecção com IBCB 121. Porém, esses valores, quando comparados com a patogenicidade dos mesmos aos pulgões Aphis gossipy (Glover, 1877) (Hemiptera, Aphididae) e Myzus persicae (Sulzer, 1776) (Hemiptera, Aphididae) demostraram certo grau de seletividade em concentrações menores (LOUREIRO et al., 2006).

Tabela 1 - Média da mortalidade de C. flavipes submetidos à exposição de diferentes isolados de Metarhiziumanisoplie nos dias após a aplicação (DAA) em laboratório.

\begin{tabular}{|c|c|c|c|c|c|c|c|c|c|c|c|c|c|}
\hline \multirow{2}{*}{\multicolumn{2}{|c|}{ Tratamentos }} & \multicolumn{12}{|c|}{ \% de redução (DAA) } \\
\hline & & $1 \underline{a}$ & $2 \underline{a}$ & 3 a & $4 \underline{a}$ & 5 a & $6 \underline{a}$ & $7 \underline{a}$ & $8 \underline{a}$ & $9 \underline{a}$ & $10 \underline{a}$ & $11^{\mathrm{a}}$ & 12 \\
\hline \multirow{6}{*}{$\begin{array}{l}8 \\
8 \\
0\end{array}$} & $1,0 \times 10^{9}$ & 0 & 13 & 30 & 46 & 77 & 76 & 100 & 100 & 100 & 100 & 100 & 100 \\
\hline & $0,5 \times 10^{9}$ & 0 & 11 & 32 & 54 & 73 & 76 & 100 & 100 & 100 & 100 & 100 & 100 \\
\hline & $1,0 \times 10^{8}$ & 1 & 8 & 26 & 46 & 75 & 81 & 82 & 84 & 100 & 100 & 100 & 100 \\
\hline & $0,5 \times 10^{8}$ & 0 & 8 & 31 & 45 & 75 & 84 & 88 & 100 & 100 & 100 & 100 & 100 \\
\hline & $1,0 \times 10^{7}$ & 1 & 15 & 30 & 38 & 65 & 68 & 68 & 69 & 70 & 100 & 100 & 100 \\
\hline & Testemunha & 1 & 4 & 12 & 18 & 44 & 45 & 45 & 46 & 49 & 59 & 67 & 100 \\
\hline \multirow{6}{*}{ 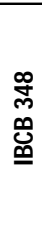 } & $1,0 \times 10^{9}$ & 7 & 11 & 27 & 40 & 73 & 75 & 84 & 91 & 93 & 100 & 100 & 100 \\
\hline & $0,5 \times 10^{9}$ & 12 & 18 & 45 & 69 & 80 & 81 & 89 & 94 & 98 & 50 & 100 & 100 \\
\hline & $1,0 \times 10^{8}$ & 11 & 18 & 59 & 87 & 92 & 96 & 100 & 100 & 100 & 100 & 100 & 100 \\
\hline & $0,5 \times 10^{8}$ & 13 & 16 & 52 & 79 & 92 & 94 & 100 & 100 & 100 & 100 & 100 & 100 \\
\hline & $1,0 \times 10^{7}$ & 9 & 14 & 54 & 74 & 94 & 100 & 100 & 100 & 100 & 100 & 100 & 100 \\
\hline & Testemunha & 0 & 12 & 23 & 31 & 40 & 43 & 43 & 43 & 46 & 46 & 47 & 100 \\
\hline \multirow{6}{*}{$\begin{array}{l}8 \\
8 \\
8 \\
5\end{array}$} & $1,0 \times 10^{9}$ & 4 & 25 & 43 & 59 & 77 & 86 & 86 & 86 & 93 & 100 & 100 & 100 \\
\hline & $0,5 \times 10^{9}$ & 4 & 32 & 45 & 58 & 69 & 80 & 86 & 92 & 96 & 100 & 100 & 100 \\
\hline & $1,0 \times 10^{8}$ & 0 & 13 & 29 & 38 & 68 & 81 & 89 & 100 & 100 & 100 & 100 & 100 \\
\hline & $0,5 \times 10^{8}$ & 3 & 10 & 13 & 35 & 67 & 88 & 94 & 97 & 100 & 100 & 100 & 100 \\
\hline & $1,0 \times 10^{7}$ & 0 & 12 & 27 & 38 & 55 & 81 & 91 & 94 & 98 & 100 & 100 & 100 \\
\hline & Testemunha & 1 & 4 & 12 & 18 & 44 & 45 & 45 & 46 & 46 & 49 & 50 & 100 \\
\hline
\end{tabular}


Observa-se ainda, que no presente estudo, as concentrações com maiores quantidades de conídios não foram aquelas que apresentaram maior porcentagem de mortalidade. A penetração dos fungos, principalmente quando utilizados em concentrações elevadas, causa o aparecimento de "orifícios" no tegumento dos insetos, os quais ficam, assim, sujeitos ao ataque de outros microrganismos (ALVES et al., 1998). Nesse caso, microrganismos oportunistas (bactérias) acabam por colonizar o corpo do hospedeiro, causando septicemia, caracterizada pelo aspecto e odor, e impedindo o crescimento do patógeno primário, ou seja, o fungo entomopatógeno interferindo nos resultados de confirmação da mortalidade do inseto.

Outro fato atrela-se a variabilidade genética entre isolados é resultado da diferença na produção de enzimas (quitinases, proteases, lípases) e toxinas, na velocidade de germinação dos conídios, na atividade mecânica de penetração na cutícula e na capacidade de colonização dos isolados (PACCOLAMEIRELLES et al., 1990).

Por meio da interpretação dos dados pode-se constatar que os isolados de M. anisopliae apresentaram baixa virulência a $C$. flavipes em condições de laboratório, uma vez que não houve a confirmação da mortalidade. Assim, ressalta-se que baixa a longevidade da $C$. flavipes pode ter influenciado nos resultados obtidos no experimento, uma vez que o adulto do parasitoide possui ciclo de vida curto (CRUZ, 2007).

\section{CONCLUSÃO}

Nas condições em que foi conduzido o experimento, pode-se concluir que os isolados de $M$. anisopliae demonstraram apresentar baixa virulência ao parasitoide larval $C$. flavipes.

\section{REFERÊNCIAS}

ALVES, S. B. Fungos entomopatogênicos. In: ALVES, S. B. (Ed.). Controle microbiano de insetos. FEALQ, 1998. Cap. 11, p. 289-381.

CONAB - COMPANHIA NACIONAL DE ABASTECIMENTO. Disponível em: http://www.conab.gov.br. Acesso 07 set. 2011.

CRUZ, I. Controle biológico de pragas na cultura de milho para produção de conservas (Minimilho), por meio de parasitóides e predadores. Sete Lagoas, MG: Embrapa Milho e Sorgo. 16p. 2007. (Circular Técnica).

DINIZ, F. R.; RODRIGUES, K. F.; ROSSI, M. M. Produção do parasitóide Cotesia flavipes (Hymenoptera: Braconidae) para controle biológico da broca da cana-de-açúcar (Diatraea saccharalis) (Lepidoptera: Crambidae) Nucleus, Edição Especial, 2008.

GOMES, F.P. Estatística experimental. 10.ed. Nobel, 1982. 430 p.

GRANCE, E.L.V.et al. Efeito de Beauveria bassiana (Bals.) Vuill. sobre o parasitóide Trichospilus Diatraeae (Cherian E Margabandhu, 1942) (Hymenoptera: Eulophidae). In: SIMPÓSIO DE CONTROLE BIOLÓGICO, 11, 2009, Bento Gonçalves, RS. Resumos... Bento Gonçalvez: 2009. CD ROOM.

JIALE, L.et al. O. Impact of Cotesia flavipes (Hymenoptera: Braconidae) as an augmentative biocontrol agent for the sugarcane borer (Lepidoptera: Crambidae) on Rice. Biological Control. v. 56, p. 159-169, 2011. 
LOUREIRO, E. S.et al. Seleção de isolados de Metarhizium anisopliae (Metch.) Sorok. Contra a cigarrinha da raiz da cana-de-açúcar Mahanarva fimbriolata (Stål, 1854) (Hemiptera: Cercopidae) em laboratório. Neotropical Entomology, v.34, p.791-798, 2005.

LOUREIRO, E. S.; MOINO JUNIOR, A. Patogenicidade de fungos hifomicetos aos pulgões Aphis gossypii Glover e Myzus persicae (Sulzer) (Hemiptera: Aphididae). Neotropical Entomology, v. 35, p. 660-665. 2006.

LOUREIRO, E. S.; MOINO JUNIOR, A. Patogenicidade de fungos entomopatogênicos a Orius insidiosus (Say) (Hemiptera: Anthocoridae). BioAssay, v.2, p.1-8, 2007.

MACEDO, D.; ALVES S. B.; VIEIRA, A. S. Screening of Metarhizium anisopliae (Metsch.) Sorok. strains against Mahanarva fimbriolata (Stål, 1854) (Hemiptera: Cercopidae). Semina: Ciências Agrárias, v.27, p 47-52. 2006.

PACCOLA-MEIRELLES, L. D.; AZEVEDO, J. L. Natural variability in the entomopathogenic fungus Beauveria bassiana. Arquivos de Biologia e Biotecnologia, v.33, p.657-672, 1990.

PINTO, A. S. Guia ilustrado de pragas da cana-de-açúcar. Piracicaba: CP2, 2009, 160 p.

SENÔ, S. S.; SANDOVAL, K. C. A. Comportamento e controle da Diatraea saccharalis na cultura da cana-de-açúcar. Nucleus, v.7, n.1, 2010. Disponível em:

http://www.nucleus.feituverava.com.br/index.php/nucleus/article/view/311/404 . DOI: 10.3738/1982.2278-311.

SILVA, J. L. C.et al. Induction of a gloverin-like antimicrobial polypeptide in the sugarcane borer Diatraea saccharalis challenged by septic injury. Brazilian Journal of Medical and Biological Research, v.43, p. 431-436, 2010.

TIAGO, P. V.et al. Differential pathogenicity of Metarhizium anisopliae and the control of the sugarcane root spittlebug Mahanarva fimbriolata. Brazilian Archives of Biology and Technology, v.54, p. 435440, 2011. 
